(C) 2004 International Press

Adv. Theor. Math. Phys. 8 (2004) 939-954

\title{
Mirror Symmetry and Supermanifold
}

\author{
Mina Aganagic and Cumrun Vafa
}

Department of Physics, University of California

366 Le Conte Hall \#7300, Berkeley, CA 94720-7300

mina@math.berkeley.edu

Physics Department, Harvard University

17 Oxford St., Cambridge, MA 02138

\begin{abstract}
We develop techniques for obtaining the mirror of Calabi-Yau supermanifolds as super Landau-Ginzburg theories. In some cases the dual can be equivalent to a geometry. We apply this to some examples. In particular we show that the mirror of the twistorial Calabi-Yau $\mathbf{C P}^{3 / 4}$ becomes equivalent to a quadric in $\mathbf{C P}^{3 \mid 3} \times \mathbf{C P}^{3 \mid 3}$ as had been recently conjectured (in the limit where the Kähler parameter of $\mathbf{C P}^{3 \mid 4}, t \rightarrow \pm \infty$ ). Moreover we show using these techniques that there is a non-trivial $\mathbf{Z}_{2}$ symmetry for the Kähler parameter, $t \rightarrow-t$, which exchanges the opposite helicity states. As another class of examples, we show that the mirror of certain weighted projective $(n+1 \mid 1)$ superspaces is equivalent to compact Calabi-Yau hypersurfaces in weighted projective $n$ space.
\end{abstract}

e-print archive:

http://lanl.arXiv.org/abs/hep-th/0403192 


\section{Introduction}

Mirror symmetry has been quite effective in clarifying non-perturbative aspects of the worldsheet theory in various contexts (see [7]). The basic result of mirror symmetry $[8]$ is that the worldsheet supersymmetric sigma model can be T-dualized to a Landau-Ginzburg theory, which in certain cases is equivalent to a supersymmetric sigma model on a different Calabi-Yau manifold.

However, early on it was realized that not every Landau-Ginzburg theory is equivalent to a Calabi-Yau manifold [17]. For example, rigid Calabi-Yau manifolds have a Landau-Ginzburg mirror, but it cannot have a CalabiYau mirror as the mirror manifold cannot have a Kähler class. To overcome this issue it was proposed in [15] that one could broaden the space of Calabi-Yau's to include Calabi-Yau supermanifolds, to restore the geometric nature of the mirror symmetry. The mirror of a Calabi-Yau may be a superCalabi-Yau. Super-Calabi-Yau manifolds were further studied in [14] where it was shown that as far as A-model topological strings are concerned certain Calabi-Yau spaces and super-Calabi-Yau spaces are equivalent. What was surprising in this context is that it was found that super-Calabi-Yau's which are weighted projective superspaces can be equivalent to complete intersections in ordinary weighted projective spaces.

Our current interest in the subject arose from the conjectures in [11]. In that paper the topological B-model on twistorial Calabi-Yau $\mathbf{C P}^{3 \mid 4}[\mathbf{1 8}]$ was mapped by a conjectured S-duality to A-model on the same CalabiYau and by a further conjectured mirror symmetry back to a B-model on a quadric in $\mathbf{C P}^{3 \mid 3} \times \mathbf{C P}^{3 \mid 3}$. The motivation for the latter mirror conjecture was that it was known that the worldsheet instantons are not needed for a stringy realization of the $\mathcal{N}=4$ supersymmetric Yang-Mills on a quadric in $\mathbf{C P}^{3 \mid 3} \times \mathbf{C P}^{3 \mid 3},[\mathbf{1 9}]$ whereas they were expected to be needed in the A-model on $\mathbf{C P}^{3 \mid 4}$. Note that these two conjectures are independent of whether one assumes the relevant instantans for the gauge sector are closed [18] or open [11] Riemann surfaces.

The S-duality conjecture has now been put on a firmer ground in [12] where it was shown that this topological S-duality is inherited for ordinary Calabi-Yau threefolds from the S-duality of type IIB superstrings in 10 dimensions as well as explaining many other existing results in the literature based on this duality. In this paper we verify the mirror conjecture of [11]. More precisely we find that the mirror of $\mathbf{C P}^{3 \mid 4}$ does depend on the Kähler class $t_{A}$ of $\mathbf{C P}^{3 \mid 4}$. We show that in the limit $t_{A} \rightarrow \pm \infty$ the mirror of $\mathbf{C P}^{3 \mid 4}$ becomes equivalent to a quadric in $\mathbf{C P}^{3 \mid 3} \times \mathbf{C P}^{3 \mid 3}$. We offer an explanation 
of why $t_{A}$ enters the picture: The value of $t_{A}$ corresponds to the expectation value of the dilaton in the $\mathcal{N}=4$ conformal supergravity. It is natural to expect that the observations of $[\mathbf{1 9}]$ could be generalized to include the more general geometry for arbitrary $t$. It is also conceivable (though we find it less appealing) that $t \rightarrow \pm \infty$ is necessary for decoupling the gravity sector from the gauge sector. At any rate for the Yang-Mills sector by itself the value of $t$ is redundant and can be reabsorbed into a redefinition of the gauge coupling constant and thus can take any value, including $t \rightarrow \pm \infty$, without affecting the amplitudes. This thus completes the circle of ideas relating perturbative Yang-Mills without instantons in the context of a quadric in $\mathbf{C P}^{3 \mid 3} \times \mathbf{C P}^{3 \mid 3}$ to its realization in twistor $\mathbf{C} \mathbf{P}^{3 \mid 4}$ which requires holomorphic instantons, as is standard by mirror symmetry.

A simple consequence of the above, is the existence of a $\mathbf{Z}_{2}$ parity symmetry which exchanges $t_{A} \leftrightarrow-t_{A}$. This symmetry turns out to exchange the two $\mathbf{C P}^{3 \mid 3}$ 's and corresponds to inverting the helicity of the fields. This is a highly non-trivial symmetry which was expected from the correspondence with Yang-Mills theory, but not manifestly realized in the twistor space $\mathbf{C P}^{3 \mid 4}$.

The method we use to prove the mirror symmetry is a natural extension of the method in $[\mathbf{8}]$ to the case of supermanifolds realized as linear sigma models with super-chiral fields. For each fermionic field we T-dualize the phase of it, which gives another bosonic mirror field. However, to conserve the central charge we also end up with two additional fermions, i.e., we still have one net fermionic dimension for the mirror. This is reminiscent of the bosonization of bosonic ghosts with first order action $[6]$.

As another application of our results we provide an alternative method to deriving the mirror symmetry for compact Calabi-Yau manifolds. The results of [14] identify the A-model on Calabi-Yau hypersurfaces with Amodel on certain weighted projective supermanifolds (without taking any hypersurface). It is easier to justify the mirror symmetry operation in this context because the circle actions are still symmetries of the theory and we can dualize them (an alternative strategy was carried out in [8]). We thus use the mirror symmetry for the non-compact supermanifold theories with $U(1)$ symmetries to rederive the mirror for ordinary compact Calabi-Yau manifolds.

The organization of this paper is as follows: In section 2, after reviewing the standard mirror symmetry derivation of $[\mathbf{8}]$ we extend it to the fermionic case. In section 3 we give a number of examples including the twistorial Calabi-Yau and the examples of [14]. In section 4 we discuss the physical implications of the twistorial mirror. 


\section{$2 \quad$ T-duality and a fermionic coordinate}

In this section we define and implement the concept of T-duality for a fermionic field. To do so we first remind the reader of how it works for the bosonic coordinates $[\mathbf{8}]$.

\subsection{Review of T-duality for bosonic coordinates}

Consider a chiral field $\Phi$ coupled to an $N=2 U(1)$ vector multiplet. Let $\Sigma$ denote the twisted chiral superfield in the $U(1)$ vector multiplet. If $\Sigma$ gets a vev, then $\Phi$ picks up mass $Q \Sigma$, where $Q$ is the $U(1)$ charge of $\Phi$. The basic strategy of $[\mathbf{8}]$ was to first view the $U(1)$ vector multiplet as a spectator sector and dualize the phase of the field $\Phi$ thus replacing $\Phi$ by a twisted chiral field $Y$. In particular we define $Y$ by the condition that

$$
|\Phi|^{2}=\operatorname{Re} Y
$$

and the condition that the phase $\theta$ of $\Phi=|\Phi| e^{i \theta}$, is T-dual to the shift $\rho$ of $Y+i \rho$ in the imaginary direction (which is periodic). As $Y$ and $\Sigma$ are both twisted chiral fields they can appear in the superpotential which is a holomorphic function of them

$$
W(\Sigma, Y)
$$

The form of $W$ is completely fixed by symmetries as follows: Since $Y$ is periodic it can only appear as a function of $\exp (-Y)$ except for the fact that $F$, the bottom component of $\Sigma$, is quantized, and thus allows a term $\Sigma Y$. In fact this term follows from the action and its coefficient is the charge of the $\Phi$ field $Q$. We thus have the term

$$
W(\Sigma, Y)=Q \Sigma Y+f(\exp (-Y))
$$

Under T-duality the momentum modes of phase of $\Phi$ are exchanged with winding modes in the imaginary direction of $Y$. For the BPS masses to agree, this means that the above LG superpotential should have critical points shifted in the imaginary part of $Y$ by $2 \pi$. Moreover the $|\Delta W|$ from one critical point to the next should be $|Q \Sigma|$ (up to some normalization conventions), which is the BPS mass of the momentum mode of $\Phi$. This uniquely fixes $f(\exp (-Y))=\exp (-Y)$. In particular we have the critical points given by

$$
\partial_{Y} W=0 \rightarrow \exp (-Y)=Q \Sigma
$$

which are at $Y_{0}+2 \pi i n$ where $\exp \left(-Y_{0}\right)=Q \Sigma$. This gives the BPS mass of the winding soliton

$$
\Delta W=2 \pi i Q \Sigma
$$


between adjacent critical points, as was required. Then the strategy in $[8]$ was to do this for every charge field $\Phi_{i}$ and then recall that $\Sigma$ is also a dynamical field. $\Sigma$ couples to the complexified (by the theta angle of the $U(1)$ gauge theory) FI term which is sometimes denoted by $t=r+i \theta$. In other words there is an additional term in the superpotential

$$
W=-t \Sigma
$$

(note for example that this leads to the theta angle term for the $U(1)$ gauge field). Thus, at the level of the superpotential, the $\Sigma$ appears only in the terms

$$
\Sigma\left(\sum_{i} Q_{i} Y_{i}-t\right) .
$$

Integrating $\Sigma$ out leads to

$$
\sum_{i} Q_{i} Y_{i}=t
$$

whose real part is the FI term condition for the vacuum

$$
\sum_{i} Q_{i}\left|\Phi_{i}\right|^{2}=r
$$

We thus end up with the superpotential

$$
W=\sum_{i} \exp \left(-Y_{i}\right)
$$

where one of the $Y_{i}$ 's can be eliminated using the constraint (2.1). This LG theory then gets related to mirror Calabi-Yau geometries.

\subsection{Extension to the super case}

Now we apply the same strategy to the case of a chiral field $\Theta$ whose top component is fermionic. We will do this presentation in two steps: First following the same steps as the bosonic case. Secondly we study the effective superpotential for $\Sigma$ and integrate in the dual fields, along the lines of mirror symmetry derivation in $[\mathbf{1}]$.

As in the bosonic case, we wish to dualize the phase of the fermionic field $\Theta$. By this we mean the phase given by rotating $\Theta$ according to

$$
\Theta \rightarrow \Theta \exp (i \omega)
$$

Clearly $\omega$ is bosonic. So if we dualize it we get a bosonic dual angle which can now be viewed as the imaginary part of a twisted chiral multiplet $X$. In particular we set, by a natural extension of the bosonic case,

$$
\Theta \Theta^{*}=-\operatorname{Re} X \text {. }
$$


Moreover the fact that momentum modes of $\Theta$ should map to winding modes of $X$ implies that winding sectors of $X$ should have mass (the choice of the sign will be explained below) $-Q \Sigma$ in other words we should have

$$
W(\Sigma, X)=-Q \Sigma X+\exp (-X) .
$$

However, introduction of one twisted chiral bosonic field cannot be the end of the story. This theory should have net dimension (i.e., central charge $\hat{c}$ ) equal to -1 , whereas $X$ has net dimension +1 . To compensate for this we should have two additional fermionic fields. Let us call them $\eta, \chi$. This is very much in the spirit of bosonization of bosonic ghost fields [6]. In order for the spectrum on the two sides to match we note that we start with the field $\Theta$ which has mass $Q \Sigma$. The only way this can be realized in the mirror theory is if one boson and one fermion cancel in pair from the partition function. For this we must have $\eta$ and $\chi$ both with mass $Q \Sigma$. This uniquely fixes the superpotential to be

$$
W(\Sigma, X, \eta, \chi)=-Q \Sigma(X-\eta \chi)+\exp (-X)
$$

Note that the critical points of $W$ with respect to $X, \eta, \chi$ give

$$
\begin{gathered}
\exp (-X)=-Q \Sigma \\
\eta=\chi=0 .
\end{gathered}
$$

At each of the critical points $X_{0}+2 \pi i n$ with $\exp \left(-X_{0}\right)=-Q \Sigma$, the excitations of $\eta$ and $\chi$ have BPS mass $-Q \Sigma$. Also the winding modes of $X$ from one critical point to another have BPS mass $-Q \Sigma$. Thus this gives back the same net spectrum as the mirror. Note that we can also shift $X \rightarrow X+\eta \chi$ and rewrite $W$ as

$$
W(\Sigma, X, \eta, \chi)=-Q \Sigma X+\exp (-X)(1+\eta \chi) .
$$

Finally, let us explain the sign of $|Q|$ in the superpotential $W$. This will also provide a consistency check on what we have done so far.

In the original theory of the fermionic chiral field $\Theta$ of charge $Q$, integrationg out of $\Theta$ generates an effective superpotential $W_{\text {eff }}(\Sigma)$ for $\Sigma$. This is generated at one loop, and as fermionic determinants tend to contribute oppositely to the bosonic determinants, the effective superpotential is the same as if we were to integrate a bosonic chiral field of charge $Q$, but of opposite sign: $W_{\text {eff }}(\Sigma)=-Q \Sigma(\log \Sigma-1)$. The same effective superpotential must be generated by starting with the dual theory and integrating out $\eta, \chi$ and $X$. The only effect of integrating out $\eta, \chi$ in (2.2) is to change the measure for $X$, so that $x=e^{-X}$ becomes a good field variable. Integrating $X$ we recover $W_{\text {eff }}(\Sigma)$ with the correct sign, as claimed.

Again just as in the bosonic case, we do this T-duality for all charged chiral fields in the theory, bosonic and fermionic, and then integrate out $\Sigma$, 
which leaves us with an effective superpotential

$$
W=\sum_{i} e^{-Y_{i}}+\sum_{j} e^{-X_{j}}\left(1+\eta_{j} \chi_{j}\right)
$$

where $Y_{i}$ 's and $X_{j}$ 's satisfy:

$$
\sum Q_{i} Y_{i}-\sum Q_{j} X_{j}=t
$$

\section{$3 \quad$ Examples}

\subsection{Calabi-Yau supermanifolds and hypersurfaces in toric vari- eties}

In our first application we show that some bosonic Calabi-Yau manifolds which are hypersurfaces in toric varieties can be equivalent to super-CalabiYau manifolds without hypersurface constraint! To be precise, the equivalence is strictly speaking only at the level of F-terms, i.e., at the level of corresponding topological field theories. Note that at the level of F-terms we can replace the fields by their constant modes, in evaluating periods. In particular as discussed in [9] the periods of a LG theory are evaluated by considering

$$
\Pi_{\alpha}=\int_{\alpha} \prod_{i} d Y_{i} \exp (-W)
$$

where $\alpha$ denotes a mid-dimensional cycle. This fact will also be useful for us when we change variables in order to identify the LG theory with a sigma model geometry.

Calabi-Yau $n$-fold $\mathcal{M}$ which is a hypersurface in toric variety is described by a theory of $n+2$ charged chiral fields $X$ and $\Phi_{i}, i=1, \ldots, n+1$ with superpotential

$$
\int d^{2} \theta X P\left(\Phi_{i}\right)
$$

The charges $Q_{i}$ of $\Phi_{i}$ and $-Q$ of $X$ satisfy

$$
\sum_{i} Q_{i}=Q
$$

and are assumed to be positive $Q, Q_{i}>0$. For the superpotential to make sense $P\left(\Phi_{i}\right)$ has to have weight $Q$. This theory has a phase where it is given by a non-linear sigma model on Calabi-Yau $Y$ which corresponds to setting

$$
P\left(\Phi_{i}\right)=0
$$


and $X=0$ in

$$
\sum_{i} Q_{i}\left|\Phi_{i}\right|^{2}-Q|X|^{2}=t
$$

modulo gauge transformations.

The claim is that the topological A-model on $\mathcal{M}$ is the same as the topological A-model on a super Calabi-Yau manifold $\hat{\mathcal{M}}$ with $n+1$ bosonic fields $\Phi_{i}$ of charge $Q_{i}$ as above, but with bosonic chiral field $X$ replaced by a fermionic chiral field $\Theta$ of charge $+Q$ and no superpotential. The corresponding D-term constraint is

$$
\sum_{i} Q_{i}\left|\Phi_{i}\right|^{2}+Q|\Theta|^{2}=t
$$

where the Kähler classes of $\mathcal{M}$ and $\hat{\mathcal{M}}$ get identified. This equivalence has been shown in [14]. In fact the idea in [14] was to use this equivalence to make the mirror symmetry of hypersurfaces more manifest, because $\hat{\mathcal{M}}$, unlike $\mathcal{M}$ does have $U(1)$ symmetries which can be T-dualized ${ }^{1}$. We will apply our machinary of mirror symmetry to $\hat{\mathcal{M}}$ and show that indeed it gives rise to the expected mirror B-model of the corresponding bosonic $\mathrm{CY}$.

The B-model mirror in case of $\hat{\mathcal{M}}$ is obtained by simple application of the above formalism. The path integral is simply given by

$$
\begin{aligned}
\hat{Z}=\int \prod_{i} d X_{i} d X d \eta d \chi \delta\left(\sum_{i} Q_{i} X_{i}-Q X-t\right) \\
\cdot \exp \left(\sum_{i} e^{-X_{i}}+e^{-X}(1+\eta \chi)\right)
\end{aligned}
$$

Integrating out $\eta, \chi$ leads just to a measure factor for $X$

$$
\hat{Z}=\int \prod_{i} d X_{i} e^{-X} d X \delta\left(\sum_{i} Q_{i} X_{i}-Q X-t\right) \exp \left(\sum_{i} e^{-X_{i}}+e^{-X}\right)
$$

Using the delta function constraint we can replace $X$ in $e^{-X}$ term in the measure by $\sum_{i} \frac{Q_{i} X_{i}}{Q}-\frac{t}{Q}$ and ignoring an irrelevant normalization $e^{-t / Q}$ we

\footnotetext{
${ }^{1}$ Note that the compactness of $\mathcal{M}$ comes from imposing $P=0$. While the A-model does not depend on the details of the superpotential $P\left(\Phi_{i}\right)$, its presence does affect the A-model theory - the central charge of the compact theory is reduced by 2 with respect to the noncompact case, which is reflected in the measure factors. The mirror for $\mathcal{M}$ was constructed from this viewpoint in $[8]$.
} 
have

$$
\begin{array}{r}
\hat{Z}=\int \prod_{i} d X_{i} e^{-\sum_{i} Q_{i} X_{i} / Q} d X \delta\left(\sum_{i} Q_{i} X_{i}-Q X-t\right) \\
\cdot \exp \left(\sum_{i} e^{-X_{i}}+e^{-X}\right)
\end{array}
$$

We can use the $\delta$ function to integrate over $X$, which just fixes its values. Defining $x_{i}=\exp \left(-Q_{i} X_{i} / Q\right)$ (which requires the standard orbifold procedure on the LG model) we obtain

$$
\hat{Z}=\int \prod_{i} d x_{i} \exp \left(\sum_{i} x_{i}^{Q / Q_{i}}+e^{t / Q} x_{1} \cdots x_{n+2}\right)
$$

which is the familiar form of the mirror LG model. The most familiar case is when $Q_{i}=1$ and $Q=n+2$. When $Q$ is not divisible by $Q_{i}$ we will have to take further field redefinitions and orbifolds to obtain a suitable geometric orbifold. We have thus obtained the mirror for $\mathcal{M}$, as was expected.

\subsection{Mirror of $\mathbf{C P}^{(3 \mid 4)}$}

The $\mathbf{C P}^{(3 \mid 4)}$ has a linear sigma model description in terms of 4 bosonic and 4 fermionic chiral fields $\Phi^{I}, \Theta^{I}, I=0, \ldots 3$ of charges +1 . Since the sums of the bosonic and fermionic charges equal, $\mathbf{C P}^{(3 \mid 4)}$ is a super CalabiYau manifold. The lowest components of the bosonic and fermionic fields describe $\mathbf{C}^{(4 \mid 4)}$, and the vacua correspond to setting D-term potential to zero

$$
\sum_{I=0}^{3}\left|\Phi^{I}\right|^{2}+\sum_{I=0}^{3}\left|\Theta^{I}\right|^{2}=r
$$

and dividing by the gauge group. The Kähler parameter ${ }^{2} r$ is complexified by the $\theta$ angle to $t=r+i \theta$.

The Landau-Ginzburg mirror of $\mathbf{C P}^{(3 \mid 4)}$ is, as discussed in the previous section

$$
\begin{aligned}
\int \prod_{I=0}^{3} d Y_{I} d X_{I} d \eta_{I} d \chi_{I} \delta\left(\sum_{I=0}^{3} Y_{I}-X_{I}-t\right) \\
\cdot \exp \left(\sum_{I=0}^{3} e^{-Y_{I}}+e^{-X_{I}}+e^{-X_{I}} \eta_{I} \chi_{I}\right)
\end{aligned}
$$

\footnotetext{
${ }^{2}$ For simplicity of notation here we denote the Kähler parameter by $t$ rather than $t_{A}$. The S-duality relates it to $t_{B} / g_{B}[\mathbf{1 2}]$ of the corresponding B-model.
} 
where

$$
\begin{gathered}
\operatorname{Re} Y_{I}=\left|\Phi^{I}\right|^{2} \\
\operatorname{Re} X_{I}=-\left|\Theta^{I}\right|^{2} .
\end{gathered}
$$

We notice that this theory has a $\mathbf{Z}_{2}$ symmetry given by

$$
\begin{aligned}
X_{I} & \leftrightarrow Y_{I} \\
\eta_{I} & \rightarrow e^{-X_{I}} \chi_{I} \\
\chi_{I} & \rightarrow e^{Y_{I}} \eta_{I} \\
t & \rightarrow-t
\end{aligned}
$$

where the change in measure factor for the $\eta_{I}, \chi_{I}$ is trivial thanks to the delta function constraint (up to a trivial normalization factor). In other words, the Kähler moduli space of the theory is half the $t$ plane. This is a highly non-trivial statement as it involves relating the non-geometric phase $t<0$ to a geometric phase $t>0$. The meaning of this $\mathbf{Z}_{2}$ symmetry will be discussed in the next section.

We want to rewrite the above as a sigma model on a hypersurface. Consider putting

$$
X_{i}=\hat{X}_{i}+Y_{0}, \quad Y_{i}=\hat{Y}_{i}+Y_{0}
$$

where $i$ runs as $i=1,2,3$. After this field redefinition, the $\delta$ function above effectively sets $X_{0}$ to

$$
X_{0}=\sum_{i=0}^{3}\left(\hat{Y}_{i}-\hat{X}_{i}\right)+Y_{0}-t .
$$

Taking all this into account and doing a further integration over the fermionic fields $\eta_{0}, \chi_{0}$ gives us:

$$
\begin{aligned}
& \int e^{-Y_{0}-\sum_{i}\left(\hat{Y}_{i}-\hat{X}_{i}\right)} d Y_{0} \prod_{i=1}^{3} d \hat{Y}_{i} d \hat{X}_{i} d \eta_{i} d \chi_{i} \\
& \quad \cdot \exp \left[e^{-Y_{0}}\left(\sum_{i=1}^{3} e^{-\hat{Y}_{i}}+e^{-\hat{X}_{i}}+1+e^{t+\sum_{i=0}^{3}\left(\hat{X}_{i}-\hat{Y}_{i}\right)}+e^{-\hat{X}_{i}} \eta_{i} \chi_{i}\right)\right] .
\end{aligned}
$$

We see that

$$
\Lambda=e^{-Y_{0}}
$$

serves as a Lagrange multiplier. We can introduce $\mathbf{C}$-valued fields $x_{i}$ and $y_{i}$, related to $\hat{X}_{i}, \hat{Y}_{i}$ by

$$
e^{-\hat{X}_{i}}=x_{i}, \quad e^{-\hat{Y}_{i}}=y_{i} x_{i}
$$

and rescale

$$
\eta_{i} \rightarrow e^{\hat{X}_{i}} \eta_{i}
$$


we find:

$$
\int d \Lambda \int \prod_{i=1}^{3} d y_{i} d x_{i} d \eta_{i} d \chi_{i} \exp \left[\Lambda\left(\sum_{i=1}^{3} x_{i} y_{i}+x_{i}+1+e^{t} y_{1} y_{2} y_{3}+\eta_{i} \chi_{i}\right)\right]
$$

Therefore, the mirror of $\mathbf{C} \mathbf{P}^{(3 \mid 4)}$ can be thought of, locally, as a super-CalabiYau hypersurface:

$$
\sum_{i=1}^{3} x_{i} y_{i}+x_{i}+1+e^{t} y_{1} y_{2} y_{3}+\eta_{i} \chi_{i}=0 .
$$

The $\mathbf{Z}_{\mathbf{2}}$ symmetry which we discussed before is not apparent in this integrated out version. However we can implement it by exchanging the roles of $X_{0}$ and $Y_{0}$ as a Lagrange multiplier, and the field we "solve" for and this leads to

$$
x_{i} \leftrightarrow y_{i}, \quad t \leftrightarrow-t .
$$

In particular in the other form we end up with the equation

$$
\sum_{i=1}^{3} x_{i} y_{i}+y_{i}+1+e^{-t} x_{1} x_{2} x_{3}+\eta_{i} \chi_{i}=0 .
$$

Note that this mirror should still enjoy the original $S U(4 \mid 4)$ symmetry of $\mathbf{C P}^{3 \mid 4}$. This is not manifest in the above form of the equation, but it is well known that some symmetries becomes less manifest upon T-dualization. For example the mirror of supersymmetric sigma model on $\mathbf{P}^{1}$ is an LG theory with

$$
W=e^{X}+e^{-X}
$$

and this does not manifestly exhibit the $S U(2)$ symmetry of $\mathbf{P}^{1}$. The same is true for the mirror of $\mathbf{C P}^{3 \mid 4}$ we have obtained here.

Now we come to the interesting part which motivated this discussion in the first place. Namely, we note that in the $t \rightarrow-\infty$ limit (3.1) is local description of a quadric in $\mathbf{C} \mathbf{P}^{(3 \mid 3)} \times \mathbf{C P}^{(3 \mid 3)}$ ! That is, up to a coordinate redefinition (3.1) becomes:

$$
\sum_{i=1}^{3} x_{i} y_{i}+1+\eta_{i} \chi_{i}=0
$$

which is an $x_{0}=1=y_{0}$ patch of a quadric

$$
\sum_{I=0}^{3} x_{I} y_{I}+\eta_{i} \chi_{i}=0
$$


in $\mathbf{C P}^{(3 \mid 3)} \times \mathbf{C P}^{(3 \mid 3)}$ with coordinates $\left(x^{I}, \eta^{i}\right)$ and $\left(y^{I}, \chi^{i}\right)$, respectively. Moreover, the $\mathbf{Z}_{\mathbf{2}}$ symmetry of the parent theory simply becomes the exchange symmetry of the two $\mathbf{C P}^{(3 \mid 3)}$ factors. We will discuss the significance of these observations in the context of twistor space description of $\mathcal{N}=4$ SYM in the following section.

\section{Interpretation for twistorial Calabi-Yau}

In the previous section we have studied the B-model mirror of the topological A-model on $\mathbf{C P}^{3 \mid 4}$. We have seen that the A-model amplitude does depend on the Kähler structure parameter $t_{A}$, as is reflected by the fact that the B-model mirror's complex structure depends on $t_{A}$. Moreover we have seen that the Kähler modulus has a $\mathbf{Z}_{\mathbf{2}}$ symmetry:

$$
t_{A} \rightarrow-t_{A}
$$

Furthermore we have shown that in the limit

$$
t_{A} \rightarrow \pm \infty
$$

the mirror geometry simplifies to a quadric in $\mathbf{C P}^{3 \mid 3} \times \mathbf{C P}^{3 \mid 3}$.

The aim of this section is twofold: First we would like to argue that the above mirror symmetry in the limit (4.2) implements the proposal of [11] in relating the two different twistorial approaches to $\mathcal{N}=4$ YangMills. Secondly we would like to give an interpretation of the $\mathbf{Z}_{2}$ symmetry (4.1). We will argue that this is equivalent to reversing the role of positive and negative helicity states, which is a-priori not manifest in the twistor approach $^{3}$, but is expected from the viewpoint of the parity symmetry of the 4 dimensional theory.

We have seen that the topological strings on $\mathbf{C P}^{3 \mid 4}$ depends on two parameters: One is the string coupling constant $g_{A}$ and the other is the Kähler class $t_{A}$ of $\mathbf{C P}^{3 \mid 4}$. We would thus expect that the corresponding gravity theory, which is expected to be $\mathcal{N}=4$ conformal supergravity [18] also has two free parameters ${ }^{4}$. Indeed the $\mathcal{N}=4$ conformal supergravity appears to have two free parameters $[\mathbf{2}, \mathbf{5}]$ :

$$
S=\int \frac{1}{\lambda^{2}}\left[\left(C_{\mu \nu \rho \sigma}\right)^{2}+S^{\prime}(\Phi, \ldots)\right]
$$

\footnotetext{
${ }^{3}$ For a proposal of how this is realized in the approach of [3] see the recent paper [4].

${ }^{4}$ We have greatly benefited from discussions with A. Tseytlin in preparation of this section.
} 
where $C$ denotes the Weyl tensor, $\Phi$ denotes a complex scalar field and $\lambda$ is the coupling constant of the theory. In principle we thus have two parameters we can control independently: $\lambda$ and $\langle\Phi\rangle$. The full structure of the Lagrangian is not known, but there is no indication that $\lambda$ can be reabsorbed into the vev of $\Phi$. In particular if one imposes an $S L(2, R)$ symmetry (whose discrete version is expected to be a true symmetry) the terms of the form $f(\Phi) C^{2}$ are ruled out [10]. Moreover the recent connections with the gauge sector induced conformal gravity in the context of AdS/CFT suggests that such terms are not generated $[\mathbf{1 0}, \mathbf{1 6}]$. This is consistent with the fact that both $g_{A}$ and $t_{A}$ deform the topological string theory. In the context of a stringy realization we would expect $\lambda$ to be identified with the string coupling constant

$$
\lambda=g_{A}
$$

and

$$
\langle\Phi-\bar{\Phi}\rangle=t_{A}
$$

This identification is also natural from the following viewpoint: It is known that we need a 2-form field in addition to the holomorphic form on the $\mathbf{C P}^{3 \mid 4}$ to give the correct gravity fields of the $\mathcal{N}=4$ conformal supergravity [20]. In [11] this field was identified with the Kähler class of $\mathbf{C P}^{3 \mid 4}$. Thus it is natural to identify the expectation value for this Kähler class with the expectation value of a field in the gravity sector and the only choice for this is the expectation value for the field $\Phi$. Why does it have to be this particular identification between $\Phi$ and $t_{A}$ ? We will now turn to answering this question.

To address this, we need to know how the $\mathcal{N}=4 \mathrm{SYM}$ couples to CSG, and in particular how the $\Phi$ couples to SYM. This has been studied in [13]. See also the recent discussion of this in $[\mathbf{1 0}]$ :

$$
S_{Y M}=\int \frac{1}{g_{A}}\left[e^{\Phi} \operatorname{tr} F_{+} \wedge F_{+}+e^{\bar{\Phi}} \operatorname{tr} F_{-} \wedge F_{-}+\cdots\right]
$$

where the $\frac{1}{g_{A}}$ in front we obtain automatically in the string realization of the $\mathcal{N}=4 \mathrm{SYM}$ via D-branes. There is a symmetry in this theory obtained by the exchange of the two helicity states:

$$
F_{+} \leftrightarrow F_{-} \quad \Phi \leftrightarrow \bar{\Phi}
$$

In the context of the twistorial realization of this theory [18], the instantons are related to the relative strength of these two terms, i.e.,

$$
\exp \left(-t_{B} / g_{B}\right)=\exp (\bar{\Phi}-\Phi) \text {. }
$$

Given the S-duality conjecture in $[\mathbf{1 1}]$, now confirmed in $[\mathbf{1 2}]$, we thus find

$$
\exp \left(-t_{A}\right)=\exp (\bar{\Phi}-\Phi),
$$


which leads us to

$$
t_{A}=\Phi-\bar{\Phi}
$$

Thus the helicity exchange symmetry acts as

$$
t_{A} \rightarrow-t_{A}
$$

as was demonstrated in the previous section (4.1) using mirror symmetry! As discussed in the previous section this $\mathbf{Z}_{2}$ symmetry exchanges the two $\mathbf{C P}^{3 \mid 3}$ 's. However the holomorphic functions on each $\mathbf{C P}^{3 \mid 3}$ signify helicities of one kind [19]. More precisely they correspond to helicity states

$$
\begin{aligned}
1(1)+ & 3\left(\frac{1}{2}\right)+3(0)+1\left(\frac{-1}{2}\right) \\
1 & \left(\frac{1}{2}\right)+3(0)+3\left(\frac{-1}{2}\right)+1(-1)
\end{aligned}
$$

respectively. In particular exchanging the two $\mathbf{C P}^{3 \mid 3}$ exchanges the +1 helicity state with the -1 helicity state. Thus we see that the (4.1) which is also accompanied with exchanging the two $\mathbf{C P}^{3 \mid 3}$ 's exchanges the two opposite helicity states. This thus gives a satisfactory explanation of the miraculous fact that the amplitudes of $\mathcal{N}=4$ YM do possess this symmetry which is rather non-trivial in the twistorial formulation of it.

Now we come to the question of the relation of the two twistor approaches $[\mathbf{1 8}, \mathbf{1 9}]$, which was conjectured in $[\mathbf{1 1}]$ to be related by S-duality and a mirror symmetry. We have shown they are equivalent only in the limit $t \rightarrow \pm \infty$. This is sufficient to establish their equivalence at the level of the Yang-Mills sector as the $t$ parameter is redundant in the Yang-Mills sector and can be reabsorbed to redefining the gauge coupling constant. Nevertheless it is natural to ask what happens if $t$ is finite. There are two natural scenarios: One possibility is that the approach of [19] could be generalized to any value of $t$. This we find very natural because the mirror does make sense for any value of $t$ and does possess a hidden $S U(4 \mid 4)$ symmetry for any value of $t$ and so it is hard to see what other field theory one can get on the mirror side. There is one other logical possibility: It may be that this story requires decoupling of gravity and that for this we need to take the $t \rightarrow \pm \infty$. This is logically possible because the gauge sector depends only on the combination $\tau_{Y M}=e^{\Phi} / g_{A}$, but we find it less appealing.

\section{Acknowledgments}

We would like to thank N. Berkovits, L. Motl, A. Neitzke and A. Tseytlin for helpful discussions. The research of M.A. was supported in part by DOE 
grant DE-FG02-96ER40956, by a DOE OJI Award and an Alfred P. Sloan Foundation fellowship. The research of C.V. was supported in part by NSF grants PHY-0244821 and DMS-0244464.

\section{References}

[1] M. Aganagic and C. Vafa, Perturbative Derivation of Mirror Symmetry, [arXiv:hep-th/0209138].

[2] E. Bergshoeff, M. de Roo, and B. de Wit, Extended Conformal Supergravity, Nucl. Phys. B 182 (1981), 173.

[3] N. Berkovits, An alternative string theory in twistor space for $\mathcal{N}=4$ super-Yang-Mills, [arXiv:hep-th/0402045].

[4] N. Berkovits and L. Motl, Cubic Twistorial String Field Theory, [arXiv:hep-th/0403187].

[5] E.S. Fradkin and A.A. Tseytlin, Conformal Supergravity, Phys. Rept. 119 (1985), 233.

[6] D. Friedan, E.J. Martinec, and S.H. Shenker, Conformal Invariance, Supersymmetry And String Theory, Nucl. Phys. B 271 (1986), 93.

[7] K. Hori et. al., Mirror Symmetry, Clay Mathematics Monographs, 1, AMS, Providence, RI, 2003.

[8] K. Hori and C. Vafa, Mirror symmetry, [arXiv:hep-th/0002222].

[9] K. Hori, A. Iqbal, and C. Vafa, D-branes and mirror symmetry, arXiv:hep-th/0005247.

[10] H. Liu and A.A. Tseytlin, $D=4$ super Yang-Mills, D $=5$ Gauged Supergravity, and $D=4$ Conformal Supergravity, Nucl. Phys. B 533 (1998), 88, [arXiv:hep-th/9804083].

[11] A. Neitzke and C. Vafa, $N=2$ Strings and the Twistorial Calabi-Yau, [arXiv:hep-th/0402128].

[12] N. Nekrasov, H. Ooguri, and C. Vafa S-duality and Topological Strings, arXiv:hep-th/0403167.

[13] M. de Roo and P. Wagemans, Gauge Matter Coupling In $N=4$ Supergravity, Nucl. Phys. B 262 (1985), 644.

[14] A. Schwarz, Sigma models having supermanifolds as target spaces, Lett. Math. Phys. 38 (1996), 91, [arXiv:hep-th/9506070].

[15] S. Sethi, Supermanifolds, rigid manifolds and mirror symmetry, Nucl. Phys. B 430 (1994), 31, [arXiv:hep-th/9404186].

[16] A.A. Tseytlin, On limits of superstring in $A d S(5) \times S^{* *} 5$, Theor. Math. Phys. 133 (2002), 1376 [Teor. Mat. Fiz. 133 (2002, 69], [arXiv:hepth/0201112]. 
[17] C. Vafa, Topological Mirrors and Quantum Rings, in 'Mirror symmetry I' (S.-T. Yau, ed.), 121, [arXiv:hep-th/9111017].

[18] E. Witten, Perturbative Gauge Theory as a String Theory in Twistor Space, [arXiv:hep-th/0312171].

[19] E. Witten, An Interpretation Of Classical Yang-Mills Theory, Phys. Lett. B 77 (1978), 394.

[20] E. Witten, private communication. 